$\mathrm{J}$ o u r n a l of

Mathematics

and Applications

JMA No 44, pp 75-91 (2021)

\title{
Zero-sum Games on a Product of Staircase-Function Finite Spaces
}

\author{
Vadim Romanuke
}

\begin{abstract}
A tractable method of solving zero-sum games defined on a product of staircase-function finite spaces is presented. The method is based on stacking solutions of "smaller" matrix games, each defined on an interval where the pure strategy value is constant. The stack is always possible, even when only time is discrete, so the set of pure strategy possible values can be continuous. Any combination of the solutions of the "smaller" matrix games is a solution of the initial zero-sum game..
\end{abstract}

AMS Subject Classification: 91A05, 91A50, 18F20.

Keywords and Phrases: Game theory; Payoff functional; Staircase-function strategy; Matrix game.

\section{Introduction}

Zero-sum games are pretty easy-to-understand models of processes where two sides (personified with a purpose to be referred to as persons or players) interact in struggling for optimizing the to-be-paid-or-pay situations $[14,15,8,10]$. Matrix games are the simplest zero-sum games whose optimal solutions are well-studied [15, 8]. Antagonistic games (where the game kernel is a surface, which may have also discontinuities, defined on a finite-dimensional Euclidean subspace) are more complicated as, opposed to matrix games, the optimal solution is not always determinable and feasible $[8,7$, 15]. Therefore, the best choice is to approximate such games to finite ones, which are easily rendered to matrix games [8]. Nevertheless, even a matrix game solution, if it is in mixed strategies, is not always practicable due to finite horizon of the game iterations (actions, plays, etc.) [9, 10, 11, 12].

A far more complicated case is a zero-sum game, in which the player's (pure) strategy is a function (e.g., of time). In such games, the payoff kernel is a functional

COPYRIGHT (C) by Publishing House of Rzeszów University of Technology P.O. Box 85, 35-959 Rzeszów, Poland 
[13]. This functional maps every pair of functions (pure strategies of the players) into a real value. In the case, when each of the players possesses a finite set of such function-strategies, the game might be rendered down to a matrix game $[8,10,12]$. Such rendering is impossible if the set of the player's function-strategies is either infinite or continuous.

If to break a time interval, on which the pure strategy is defined, into a set of subintervals, on which the strategy could be approximately considered constant, the game is not simplified much. The matter is the continuity of possible values of the strategy on a subinterval. The continuity might be removed also by discretization (or sampling) $[12,13,7]$. Thus, the set of function-strategies becomes finite. Therefore, an appropriate transformation of the continuous spaces of function-strategies into finite spaces is a problem of high priority.

\section{Motivation}

The case when the set of the player's pure strategies is finite seems to be too plain and not reflecting true practical problems. However, the number of factual actions of the players (in any game) is always finite. While the players may use strategies of whichever form they want, the eventual number of their actions has a natural limit $[1,10,2,4,5]$. Therefore, if the rules of a system to be game-modeled are defined and administered beforehand, the administrator is likely to define (or constrain) the form of the strategies players will use [16, 17].

In the simplest case, the player's pure strategy is a simple action whose duration is negligibly short and can be represented as just a time point. This case is exhaustively studied as matrix games $[15,8,12]$. In a more complicated case, the player's pure strategy is a function of time [13]. The time interval is usually short, although its length does not matter much $[2,10,1]$. A way to appropriately administer the players' actions is to constrain them to staircase functions whose points of discontinuities (breakpoints) have to be the same for both the players $[5,17,3]$. Along with the discrete time, possible values of the player's pure strategy should be discrete as well. Then, due to the set of the player's possible actions is finite, the game can be represented as a matrix game, in which the player's selection of a pure strategy means using a staircase (time) function on an interval whereon every pure strategy is defined. Obviously, the number of the player's pure strategies in the matrix staircase-function game grows immensely as the number of breakpoints ("stair" intervals) or/and the number of possible values of the player's pure strategy increases. For instance, if the number of intervals is 10 , and the number of possible values of the player's pure strategy is 6 , then there are

$$
6^{10}=60466176
$$

possible pure strategies (10-interval staircase functions of time) at this player. The respective matrix staircase-function game appears to be intractably gigantic: if even the other player has just two possible values of one's pure strategy, then there are

$$
2^{10}=1024
$$


possible pure strategies, and the resulting payoff matrix is of

$$
6^{10} \cdot 2^{10}=61917364224
$$

entries (more than 61.9 billion entries!). Therefore, a tractable method of solving zero-sum games defined on a product of staircase-function finite spaces should be suggested.

\section{Goal and tasks to be fulfilled}

Due to the above reasons, the goal is to develop a tractable method of solving zerosum games defined on a product of staircase-function finite spaces. For achieving the goal, the following tasks are to be fulfilled:

1. To formalize a zero-sum game, in which the players' strategies are staircase functions. In such a game, the set of the player's pure strategies is a continuum of staircase functions of time. Herein, the time can be thought of as it is discrete.

2. To discretize the set of possible values of the player's pure strategy so that the game be defined on a product of staircase-function finite spaces.

3. To formalize a method of solving zero-sum games defined on a product of staircase-function finite spaces.

4. To discuss applicability and significance of the method for the game theory, whereupon an unbiased conclusion is to be made.

\section{A zero-sum game with staircase-function strate- gies}

A zero-sum game, in which the player's pure strategy is a function of time, can be defined as follows. Let each of the players use time-varying strategies defined almost everywhere on interval $\left[t_{1} ; t_{2}\right]$ by $t_{2}>t_{1}$. Denote a strategy of the first player by $x(t)$ and a strategy of the second player by $y(t)$. These functions are presumed to be bounded, i. e.

$$
a_{\min } \leqslant x(t) \leqslant a_{\max } \text { by } a_{\min }<a_{\max }
$$

and

$$
b_{\min } \leqslant y(t) \leqslant b_{\max } \text { by } b_{\min }<b_{\max },
$$

defined almost everywhere on $\left[t_{1} ; t_{2}\right]$. Besides, the square of the function-strategy is presumed to be Lebesgue-integrable. Thus, pure strategies of the player belong to a rectangular functional space of functions of time:

$$
\begin{gathered}
X=\left\{x(t), t \in\left[t_{1} ; t_{2}\right], t_{1}<t_{2}: a_{\min } \leqslant x(t) \leqslant a_{\max } \text { by } a_{\min }<a_{\max }\right\} \subset \\
\subset \mathbb{L}_{2}\left[t_{1} ; t_{2}\right]
\end{gathered}
$$

and

$$
Y=\left\{y(t), t \in\left[t_{1} ; t_{2}\right], t_{1}<t_{2}: b_{\min } \leqslant y(t) \leqslant b_{\max } \text { by } b_{\min }<b_{\max }\right\} \subset
$$




$$
\subset \mathbb{L}_{2}\left[t_{1} ; t_{2}\right]
$$

are the sets of the players' pure strategies.

The first player's payoff in situation $\{x(t), y(t)\}$ is $K(x(t), y(t))$. The payoff is presumed to be an integral functional:

$$
K(x(t), y(t))=\int_{\left[t_{1} ; t_{2}\right]} f(x(t), y(t), t) d \mu(t),
$$

where $f(x(t), y(t), t)$ is a function of $x(t)$ and $y(t)$ explicitly including $t$. Therefore, the continuous zero-sum (two-person) game

$$
\langle\{X, Y\}, K(x(t), y(t))\rangle
$$

is defined on product

$$
X \times Y \subset \mathbb{L}_{2}\left[t_{1} ; t_{2}\right] \times \mathbb{L}_{2}\left[t_{1} ; t_{2}\right]
$$

of rectangular functional spaces (3) and (4) of players' pure strategies.

Presume that the players' pure strategies $x(t)$ and $y(t)$ in game (6) can be mapped to functions which both change their values for a finite number of times so that the optimal strategies in the new game are equivalent (i.e., are different only on a set of the null measure) to the optimal strategies in game (6) by (7). Denote by $N$ the number of intervals at which the player's pure strategy is constant, where $N \in \mathbb{N} \backslash\{1\}$. Then the player's pure strategy is a staircase function having only $N$ different values. If $\left\{\tau^{(i)}\right\}_{i=1}^{N-1}$ are time points at which the staircase-function strategy changes its value, where

$$
t_{1}=\tau^{(0)}<\tau^{(1)}<\tau^{(2)}<\ldots<\tau^{(N-1)}<\tau^{(N)}=t_{2},
$$

then

$$
x_{i}=x\left(\tau^{(i)}\right) \text { by } i=\overline{0, N}
$$

are the values of the first player's strategy, and

$$
y_{i}=y\left(\tau^{(i)}\right) \text { by } i=\overline{0, N}
$$

are the values of the second player's strategy. The staircase-function strategies are right-continuous:

$$
\lim _{\substack{\varepsilon>0 \\ \varepsilon \rightarrow 0}} x\left(\tau^{(i)}+\varepsilon\right)=x\left(\tau^{(i)}\right)
$$

and

$$
\lim _{\substack{\varepsilon>0 \\ \varepsilon \rightarrow 0}} y\left(\tau^{(i)}+\varepsilon\right)=y\left(\tau^{(i)}\right)
$$

for $i=\overline{1, N-1}$, whereas

$$
\lim _{\substack{\varepsilon>0 \\ \varepsilon \rightarrow 0}} x\left(\tau^{(i)}-\varepsilon\right) \neq x\left(\tau^{(i)}\right)
$$


and

$$
\lim _{\substack{\varepsilon>0 \\ \varepsilon \rightarrow 0}} y\left(\tau^{(i)}-\varepsilon\right) \neq y\left(\tau^{(i)}\right)
$$

for $i=\overline{1, N-1}$. As an exception,

$$
\lim _{\substack{\varepsilon>0 \\ \varepsilon \rightarrow 0}} x\left(\tau^{(N)}-\varepsilon\right)=x\left(\tau^{(N)}\right)
$$

and

$$
\lim _{\substack{\varepsilon>0 \\ \varepsilon \rightarrow 0}} y\left(\tau^{(N)}-\varepsilon\right)=y\left(\tau^{(N)}\right),
$$

so $x_{N-1}=x_{N}$ and $y_{N-1}=y_{N}$. Then constant values (9) and (10) by (8) mean that game (6) can be thought of as it is a succession of $N$ continuous games

$$
\left\langle\left\{\left[a_{\min } ; a_{\max }\right],\left[b_{\min } ; b_{\max }\right]\right\}, K\left(\alpha_{i}, \beta_{i}\right)\right\rangle
$$

defined on product

$$
\left[a_{\min } ; a_{\max }\right] \times\left[b_{\min } ; b_{\max }\right]
$$

by

$$
\begin{gathered}
\alpha_{i}=x(t) \in\left[a_{\min } ; a_{\max }\right] \text { and } \beta_{i}=y(t) \in\left[b_{\min } ; b_{\max }\right] \\
\forall t \in\left[\tau^{(i-1)} ; \tau^{(i)}\right) \text { for } i=\overline{1, N-1} \text { and } \forall t \in\left[\tau^{(N-1)} ; \tau^{(N)}\right],
\end{gathered}
$$

where the factual payoff in situation $\left\{\alpha_{i}, \beta_{i}\right\}$ is

$$
K\left(\alpha_{i}, \beta_{i}\right)=\int_{\left[\tau^{(i-1)} ; \tau^{(i)}\right)} f\left(\alpha_{i}, \beta_{i}, t\right) d \mu(t) \forall i=\overline{1, N-1}
$$

and

SO

$$
K\left(\alpha_{N}, \beta_{N}\right)=\int_{\left[\tau^{(N-1)} ; \tau^{(N)}\right]} f\left(\alpha_{N}, \beta_{N}, t\right) d \mu(t)
$$

$$
\begin{gathered}
K(x(t), y(t))= \\
=\sum_{i=1}^{N-1} \int_{\left[\tau^{(i-1)} ; \tau^{(i)}\right)} f\left(\alpha_{i}, \beta_{i}, t\right) d \mu(t)+\int_{\left[\tau^{(N-1)} ; \tau^{(N)}\right]} f\left(\alpha_{N}, \beta_{N}, t\right) d \mu(t)
\end{gathered}
$$

instead of (5). In other words, if every optimal situation in pure strategies in game (6) on product (7) by conditions (1) - (5) is of staircase functions satisfying conditions (8) - (16), then this game is equivalent to the succession of $N$ games (17) by (8) - (16) and (19). In this case game (6) can be represented by the succession of games (17). On the other hand, the equivalency can be settled after a requirement of that the players in game (6) use only their pure strategies in the form of staircase functions. 
Theorem 1. If each of $N$ games (17) by (8) - (16) and (19) has a single optimal situation in pure strategies, and game (6) on product (7) by conditions (1) - (5) is equivalent to the succession of these games, then the optimal situation in pure strategies in game (6) is single and can be determined by independently solving $N$ games (17).

Proof. First, the equivalency means that game (6) has only staircase solutions. Next, it should be proved that game (6) has a single optimal situation in pure strategies. Let $\left\{\alpha_{i}^{*}, \beta_{i}^{*}\right\}_{i=1}^{N}$ be optimal solutions in games (17) by (8)-(16) and (19). Then

$$
\begin{gathered}
\max _{\alpha_{i} \in\left[a_{\min } ; a_{\max }\right]} \min _{\beta_{i} \in\left[b_{\min } ; b_{\max }\right]} K\left(\alpha_{i}, \beta_{i}\right)= \\
=\max _{\alpha_{i} \in\left[a_{\min } ; a_{\max }\right]} \min _{\beta_{i} \in\left[b_{\min } ; b_{\max }\right]} \int_{\left[\tau^{(i-1)} ; \tau^{(i)}\right)} f\left(\alpha_{i}, \beta_{i}, t\right) d \mu(t)= \\
=\int_{\left[\tau^{(i-1)} ; \tau^{(i)}\right)} f\left(\alpha_{i}^{*}, \beta_{i}^{*}, t\right) d \mu(t)= \\
=\min _{\beta_{i} \in\left[b_{\min } ; b_{\max }\right]} \max _{\alpha_{i} \in\left[a_{\min } ; a_{\max }\right]} \int_{\left[\tau^{(i-1)} ; \tau^{(i)}\right)} f\left(\alpha_{i}, \beta_{i}, t\right) d \mu(t)= \\
=\max _{\beta_{i} \in\left[b_{\min } ; b_{\max }\right]} K\left(\alpha_{i}, \beta_{i}\right) \forall i=\overline{1, N-1}
\end{gathered}
$$

and

$$
\begin{gathered}
\max _{\alpha_{N} \in\left[a_{\min } ; a_{\max }\right]} \min _{\beta_{N} \in\left[b_{\min } ; b_{\max }\right]} K\left(\alpha_{N}, \beta_{N}\right)= \\
=\max _{\alpha_{N} \in\left[a_{\min } ; a_{\max }\right]} \min _{\beta_{N} \in\left[b_{\min } ; b_{\max }\right]} \int_{\left[\tau^{(N-1)} ; \tau^{(N)}\right]} f\left(\alpha_{N}, \beta_{N}, t\right) d \mu(t)= \\
=\int_{\beta_{N} \in\left[b_{\min } ; b_{\max }\right]} f\left(\alpha_{N}^{*}, \beta_{N}^{*}, t\right) d \mu(t)= \\
=\operatorname{mon}_{\alpha_{N} \in\left[a_{\min } ; a_{\max }\right]} \int_{\left[\tau^{(N-1)} ; \tau^{(N)}\right]} f\left(\alpha_{N}, \beta_{N}, t\right) d \mu(t)= \\
=\min _{\beta_{N} \in\left[b_{\min } ; b_{\max }\right]} \alpha_{\alpha_{N} \in\left[a_{\min } ; a_{\max }\right]} K\left(\alpha_{N}, \beta_{N}\right) .
\end{gathered}
$$

So,

$$
\begin{gathered}
\max _{x(t) \in X} \min _{y(t) \in Y} K(x(t), y(t))= \\
=\sum_{i=1}^{N-1}\left(\max _{\alpha_{i} \in\left[a_{\min } ; a_{\max }\right]} \min _{\beta_{i} \in\left[b_{\min } ; b_{\max }\right]} \int_{\left[\tau^{(i-1)} ; \tau^{(i)}\right)} f\left(\alpha_{i}, \beta_{i}, t\right) d \mu(t)\right)+
\end{gathered}
$$




$$
\begin{gathered}
\quad+\max _{\alpha_{N} \in\left[a_{\min } ; a_{\max }\right]} \min _{\beta_{N} \in\left[b_{\min } ; b_{\max }\right]\left[\tau^{(N-1)} ; \tau^{(N)}\right]} f\left(\alpha_{N}, \beta_{N}, t\right) d \mu(t)= \\
=\sum_{i=1}^{N-1} \int_{\left[\tau^{(i-1)} ; \tau^{(i)}\right)} f\left(\alpha_{i}^{*}, \beta_{i}^{*}, t\right) d \mu(t)+\int_{\left[\tau^{(N-1)} ; \tau^{(N)}\right]} f\left(\alpha_{N}^{*}, \beta_{N}^{*}, t\right) d \mu(t)= \\
=\sum_{i=1}^{N-1}\left(\min _{\beta_{i} \in\left[b_{\min } ; b_{\max }\right]} \max _{\alpha_{i} \in\left[a_{\min } ; a_{\max }\right]} \int_{\left[\tau^{(i-1)} ; \tau^{(i)}\right)} f\left(\alpha_{i}, \beta_{i}, t\right) d \mu(t)\right)+ \\
+\min _{\beta_{N} \in\left[b_{\min } ; b_{\max }\right]} f\left(\alpha_{N} \in \beta_{\left.\alpha_{\min } ; a_{\max }\right]} \int_{\left[\tau^{(N-1)} ; \tau^{(N)}\right]} f(t) d \mu(t)=\right. \\
=\min _{y(t) \in Y} \max _{x(t) \in X} K(x(t), y(t))
\end{gathered}
$$

and, therefore, the stack of successive solutions $\left\{\alpha_{i}^{*}, \beta_{i}^{*}\right\}_{i=1}^{N}$ is an optimal solution in game (6). Assume that there is another staircase solution in game (6). Then at least one game of those $N$ games (17) exists having at least two different solutions in pure strategies. This is a contradiction, which means that game (6) has a single optimal situation in pure strategies, in which the player's optimal strategy is a stack of the respective optimal strategies in games (17). As every optimal strategy is single, games (17) can be solved independently, whose solutions are stacked afterwards to form the optimal solution in game (6).

In fact, Theorem 1 claims that if the single pure-strategy solution of game (6) comprises staircase functions, and each of games (17) has a single pure-strategy solution, then the solution of game (6) can be determined in a simpler way, by solving games (17). They are solved in parallel, without caring of the succession. It is clear that an inverse assertion to Theorem 1 is correct as well. If it is only known that game (6) has a single optimal situation in pure strategies of staircase functions, then it does mean that the $N$ constant parts of the player's optimal strategy can be directly taken as the solutions of respective games (17). Obviously, Theorem 1 can be generalized as follows.

Theorem 2. If each of $N$ games (17) by (8) - (16) and (19) has a nonempty set of optimal situations in pure strategies, and game (6) on product (7) by conditions (1) - (5) is equivalent to the succession of these games, then every player's optimal strategy in pure strategies in game (6) is a combination of any respective $N$ constant parts of the player's optimal strategy in games (17). Apart from the combination, there are no other optimal strategies in game (6).

Proof. Let $\left\{\alpha_{i}^{*}, \beta_{i}^{*}\right\}_{i=1}^{N}$ be solutions in games (17) by (8)-(16) and (19). Then (23) - (25) hold, so the stack of these solutions is an optimal solution in game (6). First, consider the case, when each of games (17) has a finite set of optimal solutions. 
Let the $i$-th game by (19) have $n_{i}$ solutions, $i=\overline{1, N}$. Then the total number of the stacked situations is $\prod_{i=1}^{N} n_{i}$. Equilibria (23) and (24) hold for every such situation, so equilibrium (25) holds as well and thus game (6) has at least $\prod_{i=1}^{N} n_{i}$ optimal solutions. As the equivalency means that game (6) has only staircase solutions, assume that it has a solution which differs from those $\prod_{i=1}^{N} n_{i}$ situations. Then at least $\exists i_{*} \in\{\overline{1, N}\}$ such that the $i_{*}$-th game has at least $n_{i_{*}}+1$ different solutions in pure strategies. This is a contradiction, which means that game (6) has exactly $\prod_{i=1}^{N} n_{i}$ optimal situations in pure strategies. The cases of infinite and continuous sets of optimal situations in pure strategies in games (17) are considered and proved analogously.

It is easy to see that, just like Theorem 1, Theorem 2 is invertible. The parts of the player's optimal strategy of respective games (17) can be taken from any optimal strategy of this player in game (6). Nevertheless, an exhaustive enumeration of all the solutions (in the case of only finite sets of optimal solutions) may be intractable as the number of games (17) or, that is the same, the number of intervals increases. For instance, if $N=10$ and $n_{i}=4 \forall i=\overline{1,10}$ then there are 1048576 solutions in game (6), whereupon it is quite hard to rank them or select the best one.

\section{Representation by a series of matrix games}

Along with discrete time intervals, players may be forced to act within a finite subset of possible values of their pure strategies. That is, these values are

$$
a_{\min }=a^{(0)}<a^{(1)}<a^{(2)}<\ldots<a^{(M-1)}<a^{(M)}=a_{\max }
$$

and

$$
b_{\min }=b^{(0)}<b^{(1)}<b^{(2)}<\ldots<b^{(Q-1)}<b^{(Q)}=b_{\max }
$$

for the first and second players, respectively $(M \in \mathbb{N}$ and $Q \in \mathbb{N})$. Then the succession of $N$ continuous games (17) by (8) - (16) and (19) becomes a succession of $N$ matrix games

$$
\left\langle\left\{\left\{a^{(m-1)}\right\}_{m=1}^{M+1},\left\{b^{(q-1)}\right\}_{q=1}^{Q+1}\right\}, \mathbf{K}_{i}\right\rangle
$$

with payoff matrices $\mathbf{K}_{i}=\left[k_{i m q}\right]_{(M+1) \times(Q+1)}$ whose elements are

$$
k_{i m q}=\int_{\left[\tau^{(i-1)} ; \tau^{(i)}\right)} f\left(a^{(m-1)}, b^{(q-1)}, t\right) d \mu(t) \text { for } i=\overline{1, N-1}
$$


and

$$
k_{N m q}=\int_{\left[\tau^{(N-1)} ; \tau^{(N)}\right]} f\left(a^{(m-1)}, b^{(q-1)}, t\right) d \mu(t) .
$$

So, if game (6) is made equivalent to a series of matrix games (or, in other words, is represented by a series of matrix games), then it is easy to see that, unlike the representation with continuous games (17) by (8) - (16) and (19), the game always has a solution (at least, in mixed strategies).

Theorem 3. If game (6) on product (7) by conditions (1)-(5) is equivalent to the succession of $N$ matrix games (28) by (29) and (30), then the game is always solved as a stack of optimal solutions in these matrix games.

Proof. A matrix game is always solved, either in pure or mixed strategies. Denote by

$$
\mathbf{P}_{i \alpha}=\left[p_{i \alpha}^{(m)}\right]_{1 \times(M+1)}
$$

and

$$
\mathbf{P}_{i \beta}=\left[p_{i \beta}^{(q)}\right]_{1 \times(Q+1)}
$$

the mixed strategies of the first and second players, respectively, in matrix game (28). The respective sets of mixed strategies of the first and second players are

$$
\mathcal{P}_{\alpha}=\left\{\mathbf{P}_{i \alpha} \in \mathbb{R}^{M+1}: p_{i \alpha}^{(m)} \geqslant 0, \sum_{m=1}^{M+1} p_{i \alpha}^{(m)}=1\right\}
$$

and

$$
\mathcal{P}_{\beta}=\left\{\mathbf{P}_{i \beta} \in \mathbb{R}^{Q+1}: p_{i \beta}^{(q)} \geqslant 0, \sum_{q=1}^{Q+1} p_{i \beta}^{(q)}=1\right\},
$$

so $\mathbf{P}_{i \alpha} \in \mathcal{P}_{\alpha}, \mathbf{P}_{i \beta} \in \mathcal{P}_{\beta}$, and $\left\{\mathbf{P}_{i \alpha}, \mathbf{P}_{i \beta}\right\}$ is a situation in this game. Let

$$
\left\{\mathbf{P}_{i \alpha}^{*}, \mathbf{P}_{i \beta}^{*}\right\}_{i=1}^{N}=\left\{\left[p_{i \alpha}^{(m) *}\right]_{1 \times(M+1)},\left[p_{i \beta}^{(q) *}\right]_{1 \times(Q+1)}\right\}_{i=1}^{N}
$$

be optimal solutions in $N$ games (28) by (29) and (30). Then

$$
\begin{gathered}
\max _{\mathbf{P}_{i \alpha} \in \mathcal{P}_{\alpha}} \min _{\mathbf{P}_{i \beta} \in \mathcal{P}_{\beta}} \mathbf{P}_{i \alpha} \cdot \mathbf{K}_{i} \cdot \mathbf{P}_{i \beta}^{\mathrm{T}}= \\
=\max _{\mathbf{P}_{i \alpha} \in \mathcal{P}_{\alpha}} \min _{\mathbf{P}_{i \beta} \in \mathcal{P}_{\beta}} \sum_{m=1}^{M+1} \sum_{q=1}^{Q+1} k_{i m q} p_{i \alpha}^{(m)} p_{i \beta}^{(q)}= \\
=\max _{\mathbf{P}_{i \alpha} \in \mathcal{P}_{\alpha}} \min _{\mathbf{P}_{i \beta} \in \mathcal{P}_{\beta}} \sum_{m=1}^{M+1} \sum_{q=1}^{Q+1} p_{i \alpha}^{(m)} p_{i \beta}^{(q)} \int_{\left[\tau^{(i-1)} ; \tau^{(i)}\right)} f\left(a^{(m-1)}, b^{(q-1)}, t\right) d \mu(t)=
\end{gathered}
$$




$$
\begin{gathered}
=\sum_{m=1}^{M+1} \sum_{q=1}^{Q+1} p_{i \alpha}^{(m) *} p_{i \beta}^{(q) *} \int_{\left[\tau^{(i-1)} ; \tau^{(i)}\right)} f\left(a^{(m-1)}, b^{(q-1)}, t\right) d \mu(t)= \\
=\mathbf{P}_{i \alpha}^{*} \cdot \mathbf{K}_{i} \cdot\left(\mathbf{P}_{i \beta}^{*}\right)^{\mathrm{T}}= \\
=\min _{\mathbf{P}_{i \beta} \in \mathcal{P}_{\beta}} \max _{\mathbf{P}_{i \alpha} \in \mathcal{P}_{\alpha}} \sum_{m=1}^{M+1} \sum_{q=1}^{Q+1} p_{i \alpha}^{(m)} p_{i \beta}^{(q)} \int_{\left[\tau^{(i-1)} ; \tau^{(i)}\right)} f\left(a^{(m-1)}, b^{(q-1)}, t\right) d \mu(t)= \\
=\min _{\mathbf{P}_{i \beta} \in \mathcal{P}_{\beta}} \max _{i \alpha} \in \sum_{\mathcal{P}_{\alpha}} \sum_{m=1}^{M+1} \sum_{q=1}^{Q+1} k_{i m q} p_{i \alpha}^{(m)} p_{i \beta}^{(q)}= \\
=\min _{\mathbf{P}_{i \beta} \in \mathcal{P}_{\beta}} \mathbf{m a x}_{i \alpha} \in \mathcal{P}_{\alpha} \\
\mathbf{P}_{i \alpha} \cdot \mathbf{K}_{i} \cdot \mathbf{P}_{i \beta}^{\mathrm{T}} \forall i=\overline{1, N-1}
\end{gathered}
$$

and

$$
\begin{aligned}
& \max _{\mathbf{P}_{N \alpha} \in \mathcal{P}_{\alpha}} \min _{\mathbf{P}_{N \beta} \in \mathcal{P}_{\beta}} \mathbf{P}_{N \alpha} \cdot \mathbf{K}_{N} \cdot \mathbf{P}_{N \beta}^{\mathrm{T}}= \\
& =\max _{\mathbf{P}_{N \alpha} \in \mathcal{P}_{\alpha}} \min _{\mathbf{P}_{N \beta} \in \mathcal{P}_{\beta}} \sum_{m=1}^{M+1} \sum_{q=1}^{Q+1} k_{N m q} p_{N \alpha}^{(m)} p_{N \beta}^{(q)}= \\
& =\max _{\mathbf{P}_{N \alpha} \in \mathcal{P}_{\alpha}} \min _{\mathbf{P}_{N \beta} \in \mathcal{P}_{\beta}} \sum_{m=1}^{M+1} \sum_{q=1}^{Q+1} p_{N \alpha}^{(m)} p_{N \beta}^{(q)} \int_{\left[\tau^{(N-1)} ; \tau^{(N)}\right]} f\left(a^{(m-1)}, b^{(q-1)}, t\right) d \mu(t)= \\
& =\sum_{m=1}^{M+1} \sum_{q=1}^{Q+1} p_{N \alpha}^{(m) *} p_{N \beta}^{(q) *} \int_{\left[\tau^{(N-1)} ; \tau^{(N)}\right]} f\left(a^{(m-1)}, b^{(q-1)}, t\right) d \mu(t)= \\
& =\mathbf{P}_{N \alpha}^{*} \cdot \mathbf{K}_{N} \cdot\left(\mathbf{P}_{N \beta}^{*}\right)^{\mathrm{T}}= \\
& =\min _{\mathbf{P}_{N \beta} \in \mathcal{P}_{\beta}} \max _{\mathbf{P}_{N \alpha} \in \mathcal{P}_{\alpha}} \sum_{m=1}^{M+1} \sum_{q=1}^{Q+1} p_{N \alpha}^{(m)} p_{N \beta}^{(q)} \int_{\left[\tau^{(N-1)} ; \tau^{(N)}\right]} f\left(a^{(m-1)}, b^{(q-1)}, t\right) d \mu(t)= \\
& =\min _{\mathbf{P}_{N \beta} \in \mathcal{P}_{\beta}} \max _{\mathbf{P}_{N \alpha} \in \mathcal{P}_{\alpha}} \sum_{m=1}^{M+1} \sum_{q=1}^{Q+1} k_{N m q} p_{N \alpha}^{(m)} p_{N \beta}^{(q)}= \\
& =\min _{\mathbf{P}_{N \beta} \in \mathcal{P}_{\beta}} \max _{\mathbf{P}_{N \alpha} \in \mathcal{P}_{\alpha}} \mathbf{P}_{N \alpha} \cdot \mathbf{K}_{N} \cdot \mathbf{P}_{N \beta}^{\mathrm{T}} .
\end{aligned}
$$

So,

$$
\max _{x(t) \in X} \min _{y(t) \in Y} K(x(t), y(t))=
$$




$$
\begin{aligned}
& =\sum_{i=1}^{N-1}\left(\max _{\mathbf{P}_{i \alpha} \in \mathcal{P}_{\alpha}} \min _{\mathbf{P}_{i \beta} \in \mathcal{P}_{\beta}} \sum_{m=1}^{M+1} \sum_{q=1}^{Q+1} p_{i \alpha}^{(m)} p_{i \beta}^{(q)} \int_{\left[\tau^{(i-1)} ; \tau^{(i)}\right)} f\left(a^{(m-1)}, b^{(q-1)}, t\right) d \mu(t)\right)+ \\
& +\max _{\mathbf{P}_{N \alpha} \in \mathcal{P}_{\alpha}} \min _{\mathbf{P}_{N \beta} \in \mathcal{P}_{\beta}} \sum_{m=1}^{M+1} \sum_{q=1}^{Q+1} p_{N \alpha}^{(m)} p_{N \beta}^{(q)} \int_{\left[\tau^{(N-1)} ; \tau^{(N)}\right]} f\left(a^{(m-1)}, b^{(q-1)}, t\right) d \mu(t)= \\
& =\sum_{i=1}^{N-1} \sum_{m=1}^{M+1} \sum_{q=1}^{Q+1} p_{i \alpha}^{(m) *} p_{i \beta}^{(q) *} \int_{\left[\tau^{(i-1)} ; \tau^{(i)}\right)} f\left(a^{(m-1)}, b^{(q-1)}, t\right) d \mu(t)+ \\
& +\sum_{m=1}^{M+1} \sum_{q=1}^{Q+1} p_{N \alpha}^{(m) *} p_{N \beta}^{(q) *} \int_{\left[\tau^{(N-1)} ; \tau^{(N)}\right]} f\left(a^{(m-1)}, b^{(q-1)}, t\right) d \mu(t)= \\
& =\sum_{i=1}^{N-1} \mathbf{P}_{i \alpha}^{*} \cdot \mathbf{K}_{i} \cdot\left(\mathbf{P}_{i \beta}^{*}\right)^{\mathrm{T}}+\mathbf{P}_{N \alpha}^{*} \cdot \mathbf{K}_{N} \cdot\left(\mathbf{P}_{N \beta}^{*}\right)^{\mathrm{T}}= \\
& =\sum_{i=1}^{N-1}\left(\min _{\mathbf{P}_{i \beta} \in \mathcal{P}_{\beta}} \max _{\mathbf{P}_{i \alpha} \in \mathcal{P}_{\alpha}} \sum_{m=1}^{M+1} \sum_{q=1}^{Q+1} p_{i \alpha}^{(m)} p_{i \beta}^{(q)} \int_{\left[\tau^{(i-1)} ; \tau^{(i)}\right)} f\left(a^{(m-1)}, b^{(q-1)}, t\right) d \mu(t)\right)+ \\
& +\min _{\mathbf{P}_{N \beta} \in \mathcal{P}_{\beta}} \max _{\mathbf{P}_{N \alpha} \in \mathcal{P}_{\alpha}} \sum_{m=1}^{M+1} \sum_{q=1}^{Q+1} p_{N \alpha}^{(m)} p_{N \beta}^{(q)} \int_{\left[\tau^{(N-1)} ; \tau^{(N)}\right]} f\left(a^{(m-1)}, b^{(q-1)}, t\right) d \mu(t)= \\
& =\min _{y(t) \in Y} \max _{x(t) \in X} K(x(t), y(t))
\end{aligned}
$$

and, therefore, the stack of successive solutions $\left\{\mathbf{P}_{i \alpha}^{*}, \mathbf{P}_{i \beta}^{*}\right\}_{i=1}^{N}$ is an optimal solution in game (6).

Obviously, owing to the solutions of the $(M+1) \times(Q+1)$ matrix games are independent, they are stacked in a way similar to how it is done by Theorem 2 . If all $N$ matrix games are solved in pure strategies, then stacking their solutions is fulfilled trivially. When there is at least an equilibrium in mixed strategies for an interval, the stacking is fulfilled as well implying that the resulting pure-mixed-strategy solution of game (6) is realized successively, interval by interval, spending the same amount of time to implement both pure strategy and mixed strategy solutions.

\section{Exemplification}

To exemplify how the suggested method solves zero-sum games defined on a product of staircase-function finite spaces, consider a case in which $t \in[0 ; 2 \pi]$, the set of pure 
strategies of the first player is

$$
X=\{x(t), t \in[0 ; 2 \pi]: 10 \leqslant x(t) \leqslant 20\} \subset \mathbb{L}_{2}[0 ; 2 \pi]
$$

and the set of pure strategies of the second player is

$$
Y=\{y(t), t \in[0 ; 2 \pi]: 10 \leqslant y(t) \leqslant 20\} \subset \mathbb{L}_{2}[0 ; 2 \pi] .
$$

The payoff functional is

$$
K(x(t), y(t))=\int_{[0 ; 2 \pi]}[\sin (x t)-\sin (y t)+\cos (0.1 x y t)] d \mu(t) .
$$

Each of the players is allowed to change its pure strategy value at time points

$$
\left\{\tau^{(i)}\right\}_{i=1}^{19}=\{0.1 \pi i\}_{i=1}^{19}
$$

Consequently, this game can be thought of as it is defined on rectangle (18), wherein the game is

$$
\left\langle\left\{\{9+m\}_{m=1}^{11},\{9+q\}_{q=1}^{11}\right\}, \mathbf{K}_{i}\right\rangle
$$

with payoff matrices $\mathbf{K}_{i}=\left[k_{i m q}\right]_{11 \times 11}$ whose elements are

$$
\begin{gathered}
k_{i m q}=\int_{[0.1 \cdot(i-1) \pi ; 0.1 \pi i)} f(9+m, 9+q, t) d \mu(t)= \\
=\int_{[0.1 \cdot(i-1) \pi ; 0.1 \pi i)}[\sin (9 t+m t)-\sin (9 t+q t)+\cos (0.1 t(9+m)(9+q))] d \mu(t) \\
\text { for } i=\overline{1,19}
\end{gathered}
$$

and

$$
\begin{gathered}
k_{20 m q}=\int_{[1.9 \pi ; 2 \pi]} f(9+m, 9+q, t) d \mu(t)= \\
=\int_{[1.9 \pi ; 2 \pi]}[\sin (9 t+m t)-\sin (9 t+q t)+\cos (0.1 t(9+m)(9+q))] d \mu(t) .
\end{gathered}
$$

The $11 \times 11$ matrix games (37) with (38) and (39) are solved in pure and mixed strategies. The stack of the 20 first player's optimal strategies in each of those 20 $11 \times 11$ matrix games is shown in Figure 1, where the solid line corresponds to an optimal pure strategy and the dotted lines correspond to nonzero-probability pure strategies in an optimal mixed strategy. Similarly, the stack of the 20 second player's optimal strategies is shown in Figure 2. The process of how the first player's payoff changes as the time goes by is presented in Figure 3 . 


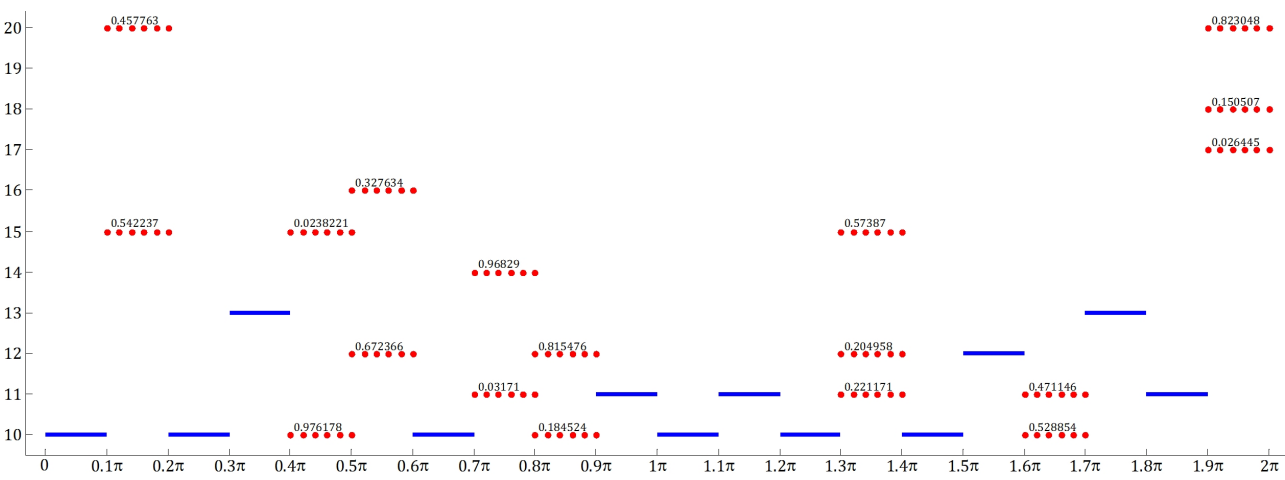

Figure 1: The stack of the 20 first player's optimal strategies

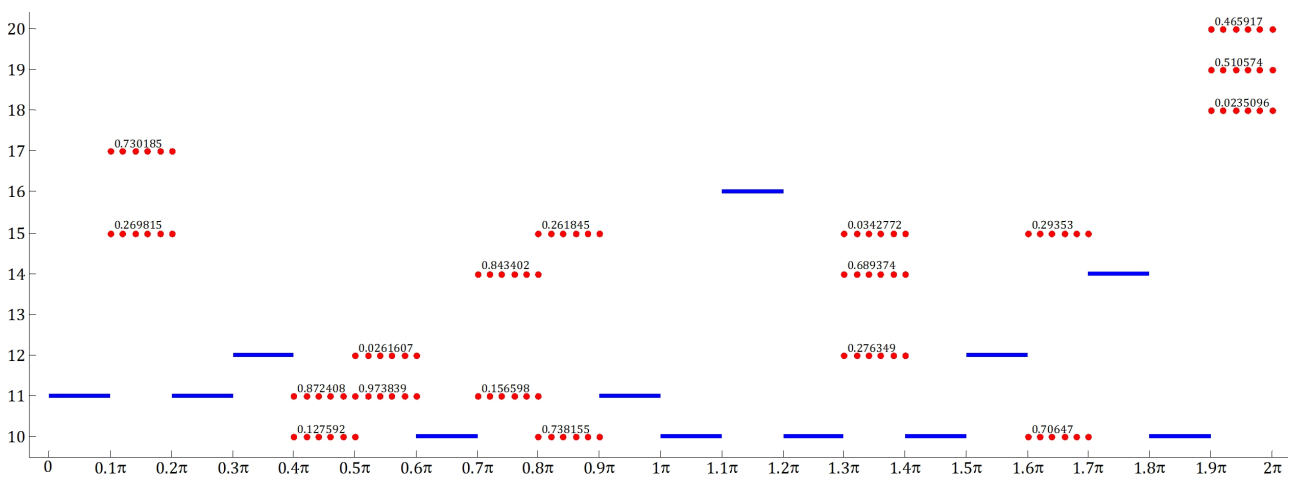

Figure 2: The stack of the 20 second player's optimal strategies

It is worth noting that the cumulative sum of the player's (either first or second) payoffs does not have to be non-decreasing (let alone increasing). This is clearly illustrated by Figure 3 as well. The first player's payoff maximum at $t=1.9 \pi$ cannot be held back from the slight decrement because in the final, 20-th (interval) game, the factual payoff of the first player is a negative amount, whereas the second player receives the positive amount. Any deviation from the optimal strategy in game (6) on product (7) by (34) - (39), say, not practicing exactly the stack in Figure 1, will not increase the cumulative sum at $t=2 \pi$ (for the first player), which is approximately 0.245389. At that, the second player cumulative loss (or payment to the first player) will not be greater than 0.245389 (if the second player sticks to the stack in Figure 2).

Another interesting peculiarity is the time of computations in this example. An $11 \times 11$ matrix game (37) with (38) and (39) is solved within 10 milliseconds on a laptop with an Intel Core i7 processor. So, the initial game is solved within 0.2 seconds 


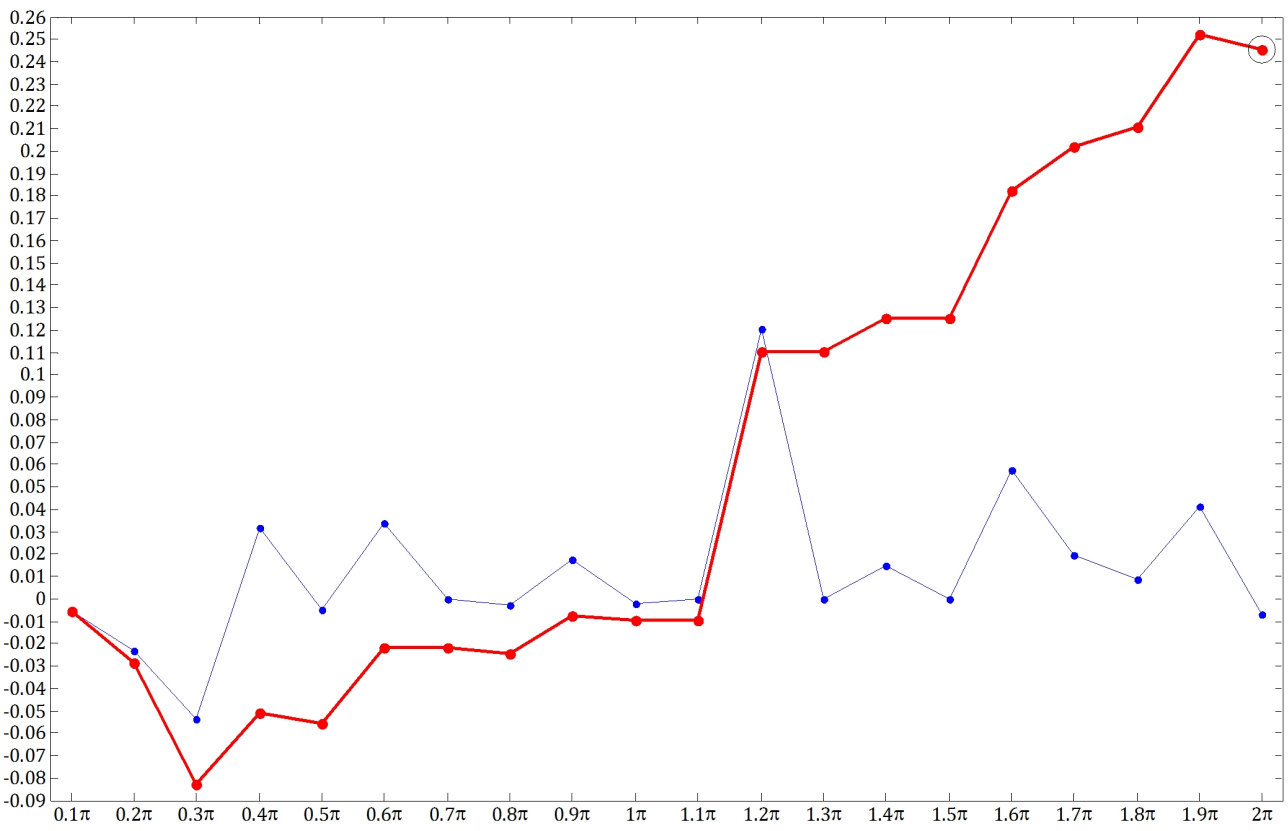

Figure 3: The first player's payoffs at the end of every interval and their cumulative sum (thicker polyline)

owing to the interval-wise solving and stacking. Surely, solving the game directly (by rendering it to the matrix game) would be quite impossible: whereas each player has $11^{20}$ pure strategies (in the form of staircase functions), the resulting $11^{20} \times 11^{20}$ matrix game cannot be solved in a reasonable time span.

\section{Discussion}

As to the computation time in general, it has an exponentially-increasing dependence on the size of the square matrix. Therefore, while a "smaller" matrix game is solved, it is presumed that the most efficient algorithm is used $[8,15]$. In a pessimistic way, the applicability of the suggested method may be limited to the "smaller" matrix game size. Solving matrix games, in which a player has a few hundred pure strategies, may be time-consuming.

A peculiar issue is how to make game (6) be equivalent to matrix games. As it has been mentioned above, the players' actions can be constrained to staircase functions whose breakpoints have to be the same for both the players. This constraint is applied by the administrator of the process modeled by game (6) on (7) by (1) - (5), but what if there is no administrator (governor, regulator, manager, etc.)? This question is still open, because no theory of self-regulating antagonistic games (in which the players would develop the strategy-generating rules fit best to the game outcome) has 
been built yet. Nevertheless, several attempts to substantiate fundamentals of such a theory have been made $[10,13,11,12,18]$.

One can notice that if time $t$ is not explicitly included into the function under the integral in (5), then, if $x(t)$ and $y(t)$ are (forcedly) set to some constants on an interval, the payoff value depends only on the length of the interval. If the length does not change, every interval has the same matrix game. The triviality of the equallength-interval solution is explained by a standstill of the players' strategies. Time variable $t$ explicitly included into (5) means that something is going on or changes within the process as time goes by (and the players develop their actions).

After all, the presented method is a significant contribution to the antagonistic game theory. It allows solving zero-sum games with staircase-function strategies in a far simpler manner. It "deeinstellungizes" the initial game along with its solution interpretation [6]. The pure-mixed-strategy solution (like that in Figure 1 and Figure 2) can be easily implemented and practiced $[9,10]$.

\section{Conclusion}

A zero-sum game defined on a product of staircase-function finite spaces is equivalent to a matrix game. However, this game payoff matrix is built very slowly, so it is impracticable to find its solution. On the other hand, the zero-sum game is equivalent to the succession of "smaller" matrix games, each defined on an interval where the pure strategy value is constant. Thus, owing to Theorem 3 , the solution of the initial game can be obtained by stacking the solutions of the "smaller" matrix games. The stack is always possible, even when only time is discrete (and the set of pure strategy possible values is continuous). Moreover, any combination of the solutions of the "smaller" matrix games is a solution of the initial zero-sum game.

Solving games on a product of staircase-function finite spaces can be studied also for the case of non-antagonistic interests of two players. Nonetheless, an approach to solving the corresponding bimatrix games is not straightforwardly deduced from the suggested method. The matter is the optimality in the matrix game does not have an analogy for the bimatrix game $[14,15]$. This specificity will make a generalized study of two-person games dissimilar to the presented study.

\section{References}

[1] M. Arblaster, Air Traffic Management. Economics, Regulation and Governance, Elsevier, 2018.

[2] M. C. Gelhausen, P. Berster, D. Wilken, Airport Capacity Constraints and Strategies for Mitigation, Academic Press, 2019.

[3] N. A. Gershenfeld, The Nature of Mathematical Modeling, Cambridge University Press, Cambridge, UK, 1999. 
[4] D. Hirshleifer, D. Jiang, Y. M. DiGiovanni, Mood beta and seasonalities in stock returns, Journal of Financial Economics 137 (1) (2020) 272-295.

[5] S. Kalaiselvam, R. Parameshwaran, Chapter 7. Seasonal Thermal Energy Storage, in: Thermal Energy Storage Technologies for Sustainability. Systems Design, Assessment and Applications, Academic Press, 2014, 145-162.

[6] F. Loesche, T. Ionescu, Mindset and Einstellung Effect, in: Encyclopedia of Creativity, Academic Press, 2020, 174-178.

[7] N. Nisan, T. Roughgarden, É. Tardos, V. V. Vazirani, Algorithmic Game Theory, Cambridge University Press, Cambridge, UK, 2007.

[8] V. V. Romanuke, Theory of Antagonistic Games, New World - 2000, Lviv, 2010.

[9] V.V. Romanuke, Convergence and estimation of the process of computer implementation of the optimality principle in matrix games with apparent play horizon, Journal of Automation and Information Sciences 45 (10) (2013) 49-56.

[10] V. V. Romanuke, Theoretic-game methods of identification of models for multistage technical control and run-in under multivariate uncertainties (a Dissertation for the Doctoral Degree of Technical Sciences in Speciality 01.05.02 Mathematical Modeling and Computational Methods), Vinnytsia National Technical University, Vinnytsia, Ukraine, 2014 (in Ukrainian).

[11] V. V. Romanuke, Approximation of unit-hypercubic infinite antagonistic game via dimension-dependent irregular samplings and reshaping the payoffs into flat matrix wherewith to solve the matrix game, Journal of Information and Organizational Sciences 38 (2) (2014) 125-143.

[12] V. V. Romanuke, Discretization of continuum antagonistic game on unit hypercube and transformation of multidimensional matrix for solving of the corresponding matrix game, Journal of Automation and Information Sciences 47 (2) (2015) 77-86.

[13] V. V. Romanuke, Finite approximation of continuous noncooperative two-person games on a product of linear strategy functional spaces, Journal of Mathematics and Applications 43 (2020) 123-138.

[14] N. N. Vorob'yov, Game Theory Fundamentals. Noncooperative Games, Nauka, Moscow, 1984 (in Russian).

[15] N. N. Vorob'yov, Game Theory for Economists-Cyberneticists, Nauka, Moscow, 1985 (in Russian).

[16] J. Yang, Y.-S. Chen, Y. Sun, H.-X. Yang, Y. Liu, Group formation in the spatial public goods game with continuous strategies, Physica A: Statistical Mechanics and its Applications 505 (2018) 737-743. 
[17] Y. Yang, J. DeFrain, A. Faruqui, Conceptual discussion on a potential hidden cross-seasonal storage: Cross-seasonal load shift in industrial sectors, The Electricity Journal 33 (8) (2020) 106846.

[18] Z. Zhou, Z. Jin, Optimal equilibrium barrier strategies for time-inconsistent dividend problems in discrete time, Insurance: Mathematics and Economics 94 (2020) 100-108.

\section{DOI: $10.7862 /$ rf.2021.6}

\section{Vadim Romanuke}

email: v.romanuke@amw.gdynia.pl

ORCID: 0000-0003-3543-3087

Faculty of Mechanical and Electrical Engineering

Polish Naval Academy

Gdynia

POLAND 